\title{
SINGULAR PERTURBATIONS FOR SYSTEMS OF LINEAR PARTIAL DIFFERENTIAL EQUATIONS
}

BY

\section{A. LIVNE AND Z. SCHUSS}

\begin{abstract}
We consider the system of linear partial differential equations $\epsilon A^{i j} u_{i j}^{\epsilon}+B^{i} u_{i}^{\epsilon}+C u^{\epsilon}=f$ where $A^{i j}, B^{i}$ are symmetric $m \times m$ matrices and $-C$ is a sufficiently large positive definite matrix. We prove that under suitable conditions $\left\|u^{\epsilon}-u\right\|_{L^{2}} \leq c \sqrt{\epsilon}\|f\|_{H^{1}}$ where $u$ is the solution of a suitable boundary value problem for the system $B^{i} u_{i}+C u=f$.
\end{abstract}

1. Introduction. This paper is concerned with the linear differential operator

$$
L_{\epsilon} u=\epsilon A^{i j} u_{i j}+B^{i} u_{i}+C u=f
$$

for $0<\epsilon<\epsilon_{0}$, where $A^{i j}, B^{i}, C$ are $m \times m$ matrix valued functions defined on a compact domain $\pi$ in $R^{n}$, whose boundary $\prod^{\circ}$ is of class $C^{\infty}$. The operator acts on vector-valued functions likewise defined on $\pi$ and subject to homogeneous boundary conditions. We assume $A^{i j}, B^{i}, C$ are symmetric or symmetrizable in the sense of [10]. We wish to compare the solution $u_{\epsilon}$ of (1.1) with the solution $v$ of the reduced problem

$$
B^{i} v_{i}+C v=f
$$

Our purpose is to find the rate of convergence of $u_{\epsilon}$ to $v$ for $\epsilon \rightarrow 0$ i.e. to derive bounds of the form $\left\|u_{\epsilon}-v\right\| \leq C \sqrt{\epsilon}\|f\|_{1}$, where $\|\cdot\|,\|\cdot\|_{1}$ are suitable norms. Recently S. V. Sivašinskii [14], C. Bardos, D. and H. Brezis [2] considered the question of strong convergence of $u_{\epsilon}$ to $v$ for similar operators. The problem of deriving bounds on the rate of convergence of singular perturbations for elliptic and parabolic equations was treated by Greenlee [5], Friedman [3], the authors [12] and others. In [5] and [3] a single elliptic or parabolic equation of higher order degenerates into a lower order elliptic or parabolic equation and the rate of convergence of $u_{\epsilon}$ to $v$ is obtained. In [12] we considered the case of a single second order equation with nonnegative characteristic form that degenerates into

Received by the editors November 16, 1972 and, in revised form, May 13, 1973.

AMS (MOS) subject classifications (1970). Primary 35F15, 35B25; Secondary 35B25, $35 \mathrm{~L} 40,35 \mathrm{~B} 30$. gence.

Key words and phrases. First order system, singular perturbations, rate of conver 
a first order equation. The methods developed in [12] are used here for systems of equations. The asymptotic nature of the solution as $\epsilon \rightarrow 0$ was thoroughly investigated by Vishik and Liusternik [15], Oleinik [13] and others. Equation (1.1) is frequently used as an "elliptic regularization" of (1.2), e.g. by Kohn and Nirenberg [7], [8] and many other authors. They prove weak convergence in a suitable space of some sequence $u_{\epsilon_{n}}$ to a solution $v$ of (1.2). However, strong convergence and its rate are not discussed. The problem of determining the boundary conditions satisfied by the limit function $v$ was discussed by Levinson [11], Ladyzhenskaya [9], Kamenomostskaya [6], SivaSinskii [14], Bardos and Brezis [2], the authors [12] and others. It is worthwhile to note that no maximum principle holds for the problem under consideration, unlike the situation in [6], [8], [9], [11], [12], [13]. We wish to thank A. Friedman and H. Brezis for bringing the problem to our attention, and to $C$. Bardos for considerably simplifying the proof of our last theorem.

2. Preliminary results and notation. Let $\mathbb{N}$ be a compact domain in $R^{n}$ as above and let $x=\left(x^{1}, \ldots, x^{n}\right)$ represent the coordinates. We use subscripts to denote differentiation, and also use the summation convention. The coefficients are real and of class $C^{\infty}$ in $\bar{\pi}$, the closure of $\pi$. We use $\nu^{j}$ to denote the $j$ th component of the unit exterior normal at $\dot{\pi}$. The boundary matrix $B_{\nu}(x)$ is defined by

$$
B_{\nu}=\sum_{j=1}^{n} B^{j} \nu^{j}
$$

We make the following assumptions.

(a) There exists a positive constant a sucb tbat for any $\xi \in R^{n},|\xi|=1$, and any $\eta \in R^{m}$

$$
\left(A^{i j} \xi^{i} \xi^{j} \eta, \eta\right) \geq \alpha|\eta|^{2}
$$

Here $($,$) denotes the scalar product in the relevant Euclidean space, |\cdot|$ denotes the Euclidean norm.

We assume that the boundary $M^{\circ}$ consists of two disjoint parts $\Sigma_{1}$ and $\Sigma_{2}$ such that

(b) The boundary matrix $B_{\nu}$ is negative definite on $\Sigma_{1}$ and positive definite on $\Sigma_{2}$, i.e. there exists a positive constant $\beta$ sucb that for any $\eta \in R^{m}$

$$
\begin{gathered}
-\left(B_{\nu} \eta, \eta\right) \geq \beta|\eta|^{2} \text { on } \Sigma_{1}, \\
\left(B_{\nu} \eta, \eta\right) \geq \beta|\eta|^{2} \text { on } \Sigma_{2} .
\end{gathered}
$$


(c) The matrix - $C$ is sufficiently large positive definite, i.e. there exists a sufficiently large positive constant $\gamma$ (independent of $\epsilon$ ) sucb that, for any $\eta \in R^{m}$, $-(C \eta, \eta) \geq 2 y|\eta|^{2}$. We use

$$
\langle u, v\rangle=\int_{\Pi}(u, v) d V, \quad\langle u, v\rangle_{\dot{M}}=\int_{\dot{M}}(u, v) d s, \quad\|u\|^{2}=\langle u, u\rangle,
$$

where $d V$ and $d s$ are the volume elements on $\pi$ and $M^{\circ}$ respectively. Similarly, $\|u\|_{k}^{\Omega}$ denotes the norm of $u(x)$ in $\left(H^{k}(\Omega)\right)^{m}$ for any subdomain $\Omega \subset \pi$ (cf. [1]).

If conditions (a), (b), (c) are satisfied then it can be shown (cf. [1, Lemma 2.1]) that there exists $\epsilon_{0}$ such that for all $\left.\left.\epsilon_{0}\right\rangle \epsilon\right\rangle 0\left\langle-L_{\epsilon} u, u\right\rangle \geq \gamma\|u\|^{2}$ for all $u \in\left(C^{\infty} \overline{J(T))^{m}}\right.$ satisfying $u=0$ on $\Sigma_{2}$, and $\partial u / \partial \nu=0$ on $\Sigma_{1}$. It follows [7] that for any $f \in\left(L_{2}(\pi)\right)^{m}$ the mixed boundary value problem

$$
L_{\epsilon} u=f \text { in } \pi \quad(\epsilon>0) \text {, }
$$

$$
\begin{array}{r}
\partial u / \partial \nu=0 \text { on } \Sigma_{1}, \\
u=0 \text { on } \Sigma_{2},
\end{array}
$$

has a unique solution $u \in\left(H^{2}(\pi)\right)^{m}$, satisfying the boundary conditions in the strong sense [1] and $y\|u\| \leq\|f\|$. If $f \in\left(H^{k}(\pi)\right)^{m}$ then $u \in\left(H^{k+2}(\pi)\right)^{m}(k \geq 1)$. Following [8] we introduce local coordinates near $\dot{\Pi}^{\circ}$ : Let $-y$ denote the distance from the boundary; on the surfaces $y=$ const, we use $x^{\prime}=\left(x^{1}, \cdots, x^{n-1}\right)$ as local coordinates, taking $x^{n}=y$ after a change of coordinates. In such coordinates $\left(x^{\prime}, y\right)$ we denote the leading coefficients of $L_{\epsilon}$ by $\epsilon A^{i j}$ for $i, j=1, \cdots$, $n-1$; otherwise we set $A^{n n}=A, 2 A^{n i}=A^{i}$ for $i \neq n$, and $B^{n}=B$, so that $L_{\epsilon}$ takes the form

$$
L_{\epsilon} u=\epsilon A u_{y y}+B u_{y}+\epsilon A^{i} u_{i y}+\epsilon A^{i j} u_{i j}+B^{i} u_{i}+C u \text {. }
$$

Here summation over $i, j$ extends from 1 to $n-1$; the subscripts $i, j, y$ denote differentiation with respect to $x^{i}, x^{j}, i, j<n$, and $y$. To be more precise, let $x_{0} \in \dot{\pi}$ and let $y=\phi(x)$ be the distance of a point $x$, in a neighborhood of $x_{0}$, to $\dot{\pi}$ so that $\phi(x)<0$ in $\pi$, and $\phi(x)>0$ outside $\pi$. We have $\phi=0$ on $\pi$ and $\nabla \phi\left(x_{0}\right) \neq 0$; setting $\xi^{i}=x^{i}, i<n, \xi^{n}=y=\phi(x)$ we have, locally, the following formulas for the coefficients of $L_{\epsilon}$ :

$$
\begin{array}{ll}
A=A^{i j} \phi^{i} \phi^{j} & (i, j=1, \ldots, n), \\
A^{i}=2 A^{i j} \phi_{j} & (j=1, \ldots, n ; i=1, \ldots, n-1), \\
B=\epsilon A^{i j} \phi_{i j}+B^{i} \phi_{i} & (i, j=1, \ldots, n) .
\end{array}
$$

Note that $B$ is positive definite near $\Sigma_{2}$ and negative definite near $\Sigma_{1}$ if $\epsilon$ is sufficiently small. 
3. A priori estimates.

Lemma 3.1. Assume (a), (b), (c) bold; let $f \in\left(H^{1}(\pi)\right)^{m}$, and $u=u_{\epsilon}(x)$ be the solution of (2.3). Then there exist neigbborboods $\pi_{2}$ and $\mathbb{Q}_{2}$ of $\Sigma_{2}$ (in $\bar{\pi}$ ) sucb that $\overline{\mathbb{M}}_{2} \subset \mathbb{Q}_{2}$, and constants $c$ (independent of $\epsilon, \gamma$ ) and $K$ (independent of $\epsilon$ ) such that

$$
\gamma^{1 / 2}\|u\|_{1}^{\pi_{2}} \leq c\|u\|_{1}^{\mathbb{Q}_{2}}+K\|f\|_{1}^{\mathbb{Q}_{2}} \text {. }
$$

Proof. The boundary $\Sigma_{2}$ may be covered by a finite number, say $k$, of small neighborhoods $U_{j}$, each of which may be described in terms of our local coordinates by

$$
\left|x^{i}-x_{0}^{i}\right|<d \quad(i=1, \ldots, n-1), \quad 0 \leq-y<d,
$$

for a small constant $d$. These neighborhoods may be chosen in such a way that any one of the enlarged regions

$$
U_{j}^{\prime}:\left|x^{i}-x_{0}^{i}\right|<2 d \quad(i=1, \ldots, n-1), \quad 0 \leq-y<2 d,
$$

intersects at most $3^{n-1}$ of the other $U_{i}^{\prime}$. We may assume that, for $d$ suitably small, the changes from coordinates on $U_{j}^{\prime}$ to coordinates on any one of the other $U_{i}^{\prime}$ have Jacobians $\geq 1 / 2$ on $U_{j}^{\prime} \cap U_{i}^{\prime}$. We define

$$
\pi_{2}=\bigcup_{j=1}^{k} U_{j}, \quad \mathbb{Q}_{2}=\bigcup_{j=1}^{k} U_{j}^{\prime} .
$$

Using local coordinates in each $U_{j}$, we define (cf. [8])

$$
\kappa=\max _{j} \int_{U_{j}} \sum_{i=1}^{n-1}\left|u_{i}\right|^{2} d V .
$$

All terms which are bounded by $c\|u\|_{1}^{Q_{2}-\pi_{2}}+K\|f\|_{1}^{Q_{2}}$ will be denoted by $H^{1 / 2}$, where $c$ denotes any constant independent of $\epsilon, \gamma$ and $K$ is any constant independent of $\epsilon$. Our aim is to show that

$$
\kappa \leq H
$$

Let $U, U^{\prime}$ be the neighborhoods where the maximum in (3.1) is achieved. We start by showing that $\left(\left\|u_{y}\right\|^{\pi_{2}}\right)^{2} \leq c k+H$. Let $\zeta$ be a nonnegative function in $C^{\infty} \bar{M}$ ) such that (i) $\zeta=1$ in $U$, (ii) $\zeta$ is independent of $y$ in $U^{\prime} \cap\{0 \leq-y<d\}$, (iii) $\zeta=0$ outside $U^{\prime}$, and (iv)

$$
\left|\zeta_{i}\right| \leq c / d
$$


We take the scalar product of (2.4) with $\zeta^{2} u_{y}$ to obtain

$$
\epsilon\left\langle A u_{y y}, \zeta^{2} u_{y}\right\rangle+\left\langle B \zeta u_{y}, \zeta u_{y}\right\rangle=-\sum_{j=0}^{4} I_{j}
$$

Clearly

$$
\left|I_{0}\right|=\left|\left\langle f, \zeta^{2} u_{y}\right\rangle\right| \leq H+\mu\left\|\zeta u_{y}\right\|^{2}, \quad\left|I_{1}\right|=\left|\left\langle C u, \zeta^{2} u_{y}\right\rangle\right| \leq H
$$

where $\mu$ is a small constant.

We have used here the estimate $\gamma\|u\| \leq\|f\|$ and integration by parts. Now let $U_{i}^{\prime}, U_{j}^{\prime}$ be any two neighborhoods such that $U_{i}^{\prime} \cap U_{j}^{\prime} \neq \varnothing$. Next, let $\left(x^{1}, \cdots, x^{n-1}\right)$ and $\left(\xi^{1}, \cdots, \xi^{n-1}\right)$ be local tangential coordinates in $U_{i}^{\prime}$ and $U_{j}^{\prime}$ respectively. If $P$ is any point in $U_{i}^{\prime} \cap U_{j}^{\prime}$ then

$$
\frac{\partial u(P)}{\partial \xi^{j}}=\sum_{i=1}^{n-1} J^{i j}(P) \frac{\partial u(P)}{\partial x^{i}}+J^{y j} \frac{\partial u(P)}{\partial y}
$$

where $J(P)$ is the Jacobian matrix at $P$. We can choose $d$ sufficiently small so that $J^{y j}(P)$ is small. Thus, for any $\mu^{\prime}>0, d$ is chosen so small that

$$
\begin{aligned}
\int_{U_{i}^{\prime} \cap U_{j}^{\prime}} \sum_{j=1}^{n-1}\left|\frac{\partial u}{\partial x^{j}}\right|^{2} d V_{x} \leq & c\left(\mu^{\prime}\right) \int_{U_{i}^{\prime} \cap U_{j}^{\prime}} \sum_{j=1}^{n-1}\left|\frac{\partial u}{\partial \xi^{j}}\right|^{2} d V_{\xi} \\
& +\mu^{\prime} \int_{U_{i}^{\prime} \cap U_{j}^{\prime}}\left|u_{y}\right|^{2} d V .
\end{aligned}
$$

Next we define $U^{\prime \prime}=U^{\prime} \cap\left(\mathscr{Q}_{2}-U\right)$. Then, using (3.6), we find that for $\mu, \mu^{\prime}$ sufficiently small

$$
\begin{aligned}
\left|I_{2}\right| & =\left|\left\langle B^{i} u_{i}, \zeta^{2} u_{y}\right\rangle\right| \leq \mu\left\|\zeta u_{y}\right\|^{2}+\mu^{\prime}\left(\left\|\zeta u_{y}\right\|^{U^{\prime \prime}}\right)^{2}+c(\mu)_{\kappa} . \\
I_{3} & =\epsilon\left\langle A^{i j} u_{i j}, \zeta^{2} u_{y}:\right. \\
& =-\epsilon\left\langle A^{i j} u_{i}, \zeta^{2} u_{y j}\right\rangle-\epsilon\left\langle A_{j}^{i j} \zeta u_{i}, \zeta u_{y}\right\rangle-2 \epsilon\left\langle A^{i j} \zeta_{j} u_{i}, \zeta u_{y}\right\rangle=J_{1}+J_{2}+J_{3} .
\end{aligned}
$$

$J_{2}$ may be estimated as in (3.5).

Next, we estimate $J_{1}$,

$$
\left|J_{1}\right|=1 / 2 \epsilon\left|\left\langle\left(A^{i j} \zeta^{2}\right)_{y} u_{i}, u_{j}\right\rangle\right| \leq c \kappa+H+\mu^{\prime}\left(\left\|\zeta u_{y}\right\|^{U^{\prime \prime}}\right)^{2} .
$$

Next, to estimate $J_{3}$, we use (3.3) and get

$$
\left|J_{3}\right| \leq \mu\left\|\zeta u_{y}\right\|^{2}+\left(\epsilon^{2} c(\mu) / d^{2}+\mu^{\prime}\right)\left(\left(\left\|u_{y}\right\|^{U^{\prime \prime}}\right)^{2}+c(\mu)_{\kappa}+H\right) .
$$


Finally

$$
\left|I_{4}\right|=\epsilon\left|\left\langle A^{i} u_{i y}, \zeta^{2} u_{y}\right\rangle\right|=1 / 2 \epsilon\left|\left\langle\left(A^{i} \zeta^{2}\right)_{i} u_{y}, u_{y}\right\rangle\right| \leq(c \epsilon / d)\left\|\zeta^{1 / 2} u_{y}\right\|^{2} .
$$

Regarding the left-hand side of (3.4) we have

$$
\epsilon\left\langle A u_{y y}, \zeta^{2} u_{y}\right\rangle \geq 1 / 2 \epsilon\left\langle A \zeta u_{y}, \zeta u_{y}\right\rangle_{i}-\epsilon\left(c\left\|\zeta u_{y}\right\|^{2}+H\right)
$$

where the first term on the right-hand side is nonnegative. Now, since $B$ is positive definite in $\mathbb{C}_{2}$ we have, upon using (3.4), (3.5), (3.8), and (3.9),

$$
\left(\left\|u_{y}\right\|^{U}\right)^{2} \leq\left(\mu^{\prime}+\mu+\epsilon / d\right) c\left(\left\|u_{v}\right\|^{U^{\prime \prime}}\right)^{2}+c \kappa+H .
$$

We can replace $U$ by any $U_{j}$ in (3.10); hence, summing over $j$, we get

$$
\left(\left\|u_{y}\right\|^{m_{2}}\right)^{2} \leq\left(\mu^{\prime}+\mu+\epsilon / d\right) c\left(\left\|u_{y}\right\|^{m_{2}}\right)^{2}+c k+H .
$$

Now, if $\mu, \mu^{\prime}, \epsilon$ are sufficiently small $\left(\mu, \mu^{\prime}\right.$ independent of $\epsilon$ ) we get

$$
\left(\left\|u_{y}\right\|^{\pi_{2}}\right)^{2} \leq c K+H
$$

We shall proceed to show that

$$
\gamma \kappa \leq c\left(\left\|u_{y}\right\|^{\pi_{2}}\right)^{2}+H .
$$

Then, using (3.11) and (3.12) we get $\kappa \leq H$, if $\gamma$ is sufficientiy large (independent of $\epsilon$ ); the assertion of the lemma follows. Keeping the same notations, consider $\left\langle L_{\epsilon} u,\left(\zeta^{2} u_{j}\right)_{j}\right\rangle=\left\langle f,\left(\zeta^{2} u_{j}\right)_{j}\right\rangle_{\circ}$ We have

$$
-\left\langle C \zeta u_{j}, \zeta u_{j}\right\rangle \geq 2 \gamma \kappa \text {. }
$$

Integration by parts and the nonnegativity of $-\left\langle L_{\epsilon} u, u\right\rangle$ disposes of terms containing four derivatives, e.g. $\left\langle A^{i j} \zeta u_{k i}, \zeta u_{k j}\right\rangle \cdot$ Regarding terms containing three derivatives, e.g. $I=\left\langle B^{i} u_{i},\left(\zeta^{2} u_{j}\right)_{j}\right\rangle$, we have

$$
I=-\left\langle B_{j}^{i} \zeta u_{i}, \zeta u_{j}\right\rangle-\left\langle B^{i} \zeta u_{i j}, \zeta u_{j}\right\rangle=J_{1}+J_{2}
$$

$J_{1}$ is estimated by (3.13), while $J_{2}=1 / 2\left\langle B_{i}^{i} \zeta u_{j}, \zeta u_{j}\right\rangle+\left\langle B^{i} \zeta u_{j}, \zeta \zeta_{i} u_{j}\right\rangle$. The first summand is estimated by (3.13) and the second by $c K+\left(\left\|u_{y}\right\|^{U^{\prime \prime}}\right)^{2}$. Hence, using (3.13), we get (3.12).

Lemma 3.2. If the conditions of Lemma 3.1 are satisfied then there exist

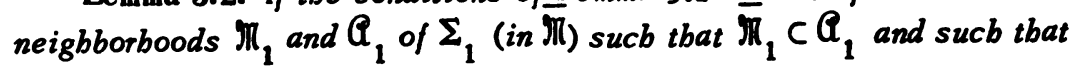

$$
\|u\|_{1}^{\pi_{1}} \leq c\|u\|_{1}^{a_{1}-\pi_{1}}+K\|f\|_{1}^{a_{1}}
$$


Proof. Let $U_{j}$ be a local coordinate neighborhood of a point in $\Sigma_{1}$ as above. Proceeding as in the proof of the previous lemma we get the estimates (3.10), (3.11), (3.13), (3.14). Next, we estimate $J_{1}$,

$$
\begin{aligned}
J_{1} & =-\left\langle\epsilon A^{i j} \zeta u_{i}, \zeta u_{y j}\right\rangle=-1 / 2\left\langle\epsilon A^{i j} \zeta u_{i}, \zeta u_{j}\right\rangle_{\Sigma_{1}}+1 / 2\left\langle\left(\epsilon A^{i j} \zeta^{2}\right)_{y} u_{i}, u_{j}\right\rangle \\
& \leq c \kappa+H+\mu^{\prime}\left(\left\|\zeta u_{y}\right\|^{\prime \prime}\right)^{2}
\end{aligned}
$$

since the boundary term is nonpositive. Multiplying (3.11) by -1 and noting that for any $\eta \in R^{m},-(B \eta, \eta) \geq 1 / 2 \beta|\eta|^{2}$ in $\mathfrak{C}_{1}$, we see that for $\epsilon, \mu, \mu^{\prime}$ sufficiently small we get

$$
\left(\left\|u_{y}\right\|^{m_{1}}\right)^{2} \leq c k+H
$$

The analogue of (3.12),

$$
\gamma \kappa \leq c\left(\left\|u_{y}\right\|^{\pi_{1}}\right)^{2}+H
$$

is obtained in a similar way.

Theorem 3.1. Assume (a), (b), (c) bold. Let $f \in\left(H^{1}(\pi)\right)^{m}$ and $u$ be the solution of (2.3). Then there exists a constant $c$, independent of $\epsilon$, sucb that

$$
\|u\|_{1} \leq c\|f\|_{1}
$$

if $\gamma$ is sufficiently large (independent of $\epsilon$ ).

Proof. Set $r_{i}=\left\{x \in \mathbb{N} \mid \operatorname{dist}\left(x, \Sigma_{i}\right)<d / 2\right\} \quad(i=1,2)$. Notice that $r_{i} \subset \pi_{i}$ $(i=1,2)$. We define

$$
\Omega=\bar{\pi}-\pi_{1}-\pi_{2}, \quad \Omega^{\prime}=\bar{\pi}-r_{1}-r_{2} .
$$

Let $\zeta$ be a smooth function defined on $\bar{\pi}$ such that $\zeta=1$ on $\Omega, \zeta=0$ outside $\Omega^{\prime}$. In view of Lemmas $3.1,3.2$ it suffices to show that

$$
\gamma^{1 / 2}\|u\|_{1}^{Q} \leq c\|u\|_{1}^{\Omega^{\prime}-\Omega}+K\|f\|_{1}
$$

This estimate is established by arguments similar to the ones used in the proof of the previous lemmas. To be more specific consider

$$
\left\langle L_{\epsilon} u_{9}\left(\zeta^{2} u_{k}\right)_{k}\right\rangle=\left\langle f_{0}\left(\zeta^{2} u_{k}\right)_{k}\right\rangle
$$

(in the usual Cartesian coordinate system). Integration by parts gives an estimate of the left-hand side by the norms of $u_{i} \zeta_{j}$, but since $\zeta_{i}=0$ in $\Omega$ we get (3.18). 
4. Singular perturbations. Let $u_{0}$ be the solution of the first order system

$$
\begin{array}{ll}
B^{i} u_{i}+C u=f & \text { in } \pi, \\
u=0 & \text { on } \Sigma_{2} .
\end{array}
$$

It is well known [7], [10] that such a solution exists, $u_{0} \in\left(H^{1}(\pi)\right)^{m}$ if $f \in\left(H^{1}(\pi)\right)^{m}$ and $\left\|u_{0}\right\|_{1} \leq c\|f\|_{1}$. The main result of this paper is

Theorem 4.1. Assume conditions (a), (b), (c) bold. Let $f \in\left(H^{1}(\pi)\right)^{m}$, then the solutions $\left\{u_{\epsilon}\right\}$ of $(2.3)$ converge in $\left(L_{2}(\pi)\right)^{m}$ to $u_{0}$, and

$$
\left\|u_{\epsilon}-u_{0}\right\| \leq c \sqrt{\epsilon}\|f\|_{1},
$$

where $c$ is a constant independent of $\epsilon$.

Proof. Denote by $\mathbf{A}$ the operator $-A^{i j} D_{i j}$ in $\left(L_{2}(\pi)\right)^{m}=H$ whose domain $D(\mathbf{A})$ is the closure of the set $\left\{v \in\left(C^{2}(\bar{\pi})\right)^{m} \mid v=0\right.$ on $\Sigma_{2}, \partial v / \partial \nu=0$ on $\left.\Sigma_{1}\right\}$ in $\left(H^{2}(\pi)\right)^{m}$. Similarly, let $\mathbf{B}$ be the operator $-\left(B^{i} D_{i}+C\right)$ in $\left(L_{2}(\pi)\right)^{m}$ whose domain $D(\mathbf{B})$ is the closure of the set $\left\{v \in\left(C^{1} \overline{(\pi)}\right)^{m} \mid v=0\right.$ on $\left.\Sigma_{2}\right\}$ in $\left(H^{1}(\pi)\right)^{m}$. Then [2] $\mathrm{A} u_{0} \in\left(H^{-1}(\pi)\right)^{m}$. Therefore

$$
\epsilon \mathbf{A}\left(u_{\epsilon}-u_{0}\right)+\mathbf{B}\left(u_{\epsilon}-u_{0}\right)=-\mathbf{A} u_{0}
$$

Now we take the scalar product of (4.3) with $u_{\epsilon}-u_{0}$ in $H^{\prime}$. Since the operator $\mathbf{B}$ is strictly positive we get

$$
\left\|u_{\epsilon}-u^{2} \leq \epsilon c\right\| u_{0}\left\|_{1}\right\| u_{\epsilon}-u_{0}\left\|_{1} \leq \epsilon c\right\| f \|_{1}^{2}
$$

by Theorem 3.1. Thus Theorem 4.1 is proved.

Remark. The boundary conditions in (4.1) are very restrictive. They follow from the restrictive conditions $(2.2),\left(2.2^{\prime}\right)$. It would be desirable to replace them by the usual conditions of [4]. The question of strong convergence of $u_{\epsilon}$ to $u_{0}$ was settled in [2] and [14] for a wide class of boundary conditions, but the rate of convergence is still unknown.

\section{REFERENCES}

1. S. Agmon, Lectures on elliptic boundary value problems, Van Nostrand Math. Studies, no. 2, Van Nostrand, Princeton, N. J., 1965. MR 31 \#25.04.

2. C. Bardos, D. Brezis and H. Brezis, Perturbations singulières et prolongements maximaux d'opérateurs positifs, Arch. Rational Mech. Anal. (to appear).

3. A. Friedman, Singular perturbations for partial differential equations, Arch. Rational Mech. Anal. 29 (1968), 289-303. MR 37 \#1754.

4. K. O. Friedrichs and P. D. Lax, Boundary value problems for first order operators, Comm. Pure Appl. Math. 18 (1965), 355-388. MR 30 \#5186. 
5. W. M. Greenlee, Rate of convergence in singular perturbations, Ann. Inst. Fourier (Grenoble) 18 (1968), fasc. 2, 135-191. MR 39 \#3133.

6. S. Kamenomostskaya, The first boundary problem for equations of elliptic type with a small parameter with the highest derivatives, Izv. Akad. Nauk SSSR Ser. Mat. 19 (1955), 345-360. (Russian) MR 17, 627.

7. J. J. Kohn and L. Nirenberg, Non-coercive boundary value problems, Comm. Pure Appl. Math. 18 (1965), 443-492. MR 31 \#6041.

8. - Degenerate elliptic-parabolic equations of second order, Comm. Pure Appl. Math. 20 (1967), 797-872. MR 38 \#2437.

9. 0. A. Ladyženskaja, On equations with small parameter in the higher derivatives in linear partial differential equations, Vestnik Leningrad. Univ. Ser. Mat. Meh. Astr. 12 (1957), no. 7, 104-120. (Russian) MR 19, 656.

10. P. D. Lax and R. S. Phillips, Local boundary conditions for dissipative symmetric linear differential operators, Comm. Pure Appl. Math. 13 (1960), 427-455. MR 22 \#9718.

11. N. Levinson, The first boundary value problem for $\epsilon \Delta u+A(x, y) u_{x}+B(x, y) u_{y}+$ $C(x, y) u=D(x, y)$ for small $\epsilon$, Ann. of Math. (2) 51 (1950), 428-445. MR 11, 439.

12. A. Livne and $Z$. Schuss, Singular perturbations for degenerate elliptic equations of second order, Arch. Rational Mech. Anal. (to appear).

13. O. A. Oleinik, On equations of elliptic type with a small parameter in the highest derivatives, Mat. Sb. 31 (73) (1952), 104-117. (Russian) MR 14, 560.

14. S. V. Sivašinskii, The introduction of 'viscosity' into first order linear symmetric systems, Vestnik Leningrad. Univ. 25 (1970), no. 19, 54-57. (Russian) MR 44 \#593.

15. M. I. Višik and L. A. Ljusternik, Regular degeneration and boundary layer for linear differential equations with a small parameter, Uspehi Mat. Nauk 12 (1957), no. 5 (77), 3-122; English transl., Amer. Math. Soc. Transl. (2) 20 (1962), 239-364. MR 20 \#2539; $25 \# 322$.

DEPARTMENT OF MATHEMATICAL SCIENCES, TEL-AVIV UNIVERSITY, TEL-AVIV, ISRAEL 